\title{
Apoyo familiar y docente percibido: valoraciones que niños, niñas y adolescentes hacen de los nuevos componentes de la calidad educativa en Chile
}

Perceived family and teacher support: assessments that children and adolescents make of the new components of educational quality in Chile

\author{
DR. CARLOS RENÉ RODRÍGUEZ-GARCÉS \\ Autor, Director Centro de Investigación CIDCIE \\ Universidad del Bío-Bío. Chillán, Chile \\ carlosro@ubiobio.cl
}

\section{GERALDO PADILLA FUENTES}

gpadilla@ubiobio.cl

HÉCTOR VARGAS MUÑOZ

hvargas@ubiobio.cl

Centro de Investigación CIDCIE

Universidad del Bío-Bío. Chillán, Chile

\section{RESUMEN}

Utilizando los datos de la Primera Encuesta de Desarrollo Humano en Niños, Niñas y Adolescentes realizada en Chile en el año 2017, se analiza la percepción que los estudiantes tienen del aula, colegio y familia a la cual pertenecen, en tanto constituyen nuevos indicadores de la calidad educativa. Al respecto, destaca la alta valoración en relación con el apoyo familiar y docente percibido. Los padres y los profesores se posicionan como figuras positivas para el alumno al brindar confort emocional y propiciar ambientes de respeto y oportunidades de expresión, que son componentes elementales para la sensación de bienestar, generación de condiciones para el aprendizaje y desarrollo de conductas prosociales. Los resultados enfatizan la necesaria imbricación familia-escuela para futuras estrategias de mejoramiento educativo.

Palabras clave: rol de la familia, coexistencia pacífica, percepción, psicología de la educación, evaluación de la educación. 


\section{ABSTRACT}

Using the data from the First Survey of Human Development in Children and Adolescents held in Chile in 2017, the perception that students have of the classroom, school and family to which they belong is analyzed, as new indicators of educational quality. Highlights the high perceived value of the family and teacher support. Parents and teachers are positioned as positive figures for the student as they provide emotional comfort and foster environments of respect and opportunities for expression, being elementary components for the sense of well-being, generation of learning conditions and development of prosocial behaviors. Results that emphasize the necessary family-school imbrication for future strategies of educational improvement.

Key words: family role, peaceful coexistence, perception, educational psychology, educational evaluation.

\section{INTRODUCCIÓN}

La convivencia escolar es uno de los temas fundamentales de las políticas educativas, pues incide tanto en el rendimiento académico como en el desarrollo psicosocial de los estudiantes; centralidad temática que ha propiciado, por parte de organismos públicos y privados, la conversión de este fenómeno en objeto de estudio e intervención.

La escuela representa una institución formal donde se establece el contacto primario, directo y prolongado de niños, niñas y adolescentes (NNA) con figuras de autoridad fuera de la familia (Gilbert, 2010), en un espacio de relaciones sociales organizadas jerárquicamente en las que se conforma un microcosmos de interacciones entre pares, educadores y su entorno (Rentería, González, García y Macías, 2013).

Los colegios, como cualquier lugar en el que conviven diferentes personas, poseen un ambiente que delimita y singulariza las interacciones, impregnándoles unas características y objetivos particulares que son parte de la "personalidad" de la institución (Moos, 1974). Eso es lo que Kurt Lewin (1939) llamó clima social, cuyos principios se utilizan para la definición y estudio del clima escolar y áulico, 
constructo que establece una relación indisoluble entre el comportamiento humano y el campo psicológico en el que las personas se desenvuelven.

Al respecto, los salones de clase son subsistemas dentro de la escuela. Allí emergen acuerdos específicos entre profesores y estudiantes que dotan al espacio de atributos intelectuales, sociales, emocionales y físicos propios, únicos por cuanto construyen marcos simbólicos que adaptan los valores institucionales (Gázquez, et al., 2009; Ambrose, Bridges, DiPietro y Lovett, 2010). En ese contexto, el rol docente y el apoyo percibido que de él tienen los estudiantes aparecen como factores relevantes en la construcción de un buen clima escolar y áulico, que sirve de sustento para garantizar la efectividad de la intervención pedagógica, los aprendizajes curriculares y la instalación de conductas prosociales y ciudadanas en el alumnado; función y responsabilidad intrínseca de la escuela (Cava y Musitu, 2002; López de Mesa et al., 2013; Rentería et al., 2013; Treviño, Place y Gempp, 2012; Paneiva, Bakker y Rubiales, 2018).

En efecto, se ha reunido suficiente evidencia sobre cómo el docente y los estímulos -oportunidades de implicación personal, valoración y apoyo que entregan a sus alumnos- afectan positivamente el clima áulico (Paneiva, Bakker y Rubiales, 2018; Treviño, Place y Gempp, 2012; Aron, Milicic y Armijo, 2012; Rodríguez, 2004).

La vivencia de emociones positivas en el aula aporta de manera significativa a la expresión de conductas favorables en la interacción con los pares, así como en la adquisición de un repertorio de habilidades que permiten enfrentar situaciones de conflicto, frustración o expresión de emociones negativas. Sin embargo, a pesar de esta evidencia, el desarrollo curricular en los establecimientos educacionales persiste en un abordaje exhaustivo de contenidos centrados en la cognición, más que en la emoción. En ese sentido, es menester que el profesor desarrolle una capacidad empática y de reconocimiento de los estados emocionales de sus alumnos, para tener así una relación cordial; competencia particularmente relevante en la etapa de la educación infantil y primaria, dada su incidencia en el aprendizaje futuro (Cassinda et al., 2017).

Por el contrario, las actitudes peyorativas y discriminatorias de los profesores y las experiencias de rechazo social vividas en la escuela estarían asociadas a: la aparición de conductas negativas, agresivas y de inestabilidad emocional; y al surgimiento y profundización de comportamientos disruptivos que deterioran el 
clima del aula y las condiciones en las que se suscita el aprendizaje (Estévez et al., 2008; Aron, Milicic y Armijo, 2012; Paneiva, Bakker y Rubiales, 2018).

En todo caso, uno de los pilares para mejorar la convivencia en los establecimientos es trabajar planes de convivencia organizados y compartidos por la comunidad escolar, orientados hacia la promoción de competencias sociales, afectivas y emocionales, en lugar de mantener discursos pedagógico-normativos estrictos (Del Rey, Ortega y Feria, 2009). Algo relevante es cómo la institución pueden hacer sentir al estudiante parte de algo mayor y lograr que estos aprendan a vivir juntos, lo que hace referencia a la necesidad de vincular subsistemas al interior de la comunidad educativa y difuminar las rígidas barreras entre profesor, estudiantes y padres, fomentando la importancia de establecer reglas y normas comunes, donde la opinión y valoración de los miembros esté asegurada; con esto, mejorarían las condiciones de educabilidad del ambiente escolar, a la vez que se entregan pautas de convivencia replicables para la vida en sociedad.

No obstante, tales argumentos, en su punto de partida, omiten la vinculación del aula con algunas variables que perfilan la dinámica familiar, los estilos parentales de crianza y el impacto que tienen estos sobre las actividades 0 comportamientos positivos que el estudiante aporta al clima social en su escuela (Estévez et al., 2008). De allí, la queja generalmente expresada por los docentes con respecto al exiguo protagonismo que los padres y apoderados asumen como agentes educativos, tanto en su casa al reforzar los aprendizajes o en la escuela al apoyar actividades o proyectos (Caballero, 2010). También, existe evidencia del efecto positivo sobre el desempeño escolar que promueven las relaciones de participación de apoderados junto a los profesores, aunque ello se relativiza en virtud del capital social y económico familiar (Gubbins et al., 2017). Tal es el caso de hogares vulnerables, en los cuales la mayoría de los padres no supera la educación básica, donde se registraría una escasa implicación parental educativa producto de la ausencia de conocimientos necesarios para apoyar los procesos de aprendizaje de sus hijos (Díaz y Osuna, 2017). Aunque, a juicio de los propios estudiantes, esta implicación no sería relevante en la calidad formativa de un colegio, más allá de la mediación en la resolución de conflictos profesor-alumno (Gázquez, Pérez y Carrión, 2011).

En su rol educativo y de desarrollo de competencias prosociales, la escuela es acompañada por la institución familiar, por cuanto profesores, compañeros y padres representan las figuras más importantes durante el período de infancia y adolescencia (Lila, Buelga y Musitu, 2006). El apoyo familiar, entendido como el 
grado de involucramiento de los padres en las actividades escolares y tiempo de acompañamiento en las tareas de sus hijos, es considerado uno de los elementos relevantes en el proceso educativo (Bazán et al., 2007; Martín et al., 2015; Céspedes, 2008; Mingo y Escudero, 2008).

Incluso, algunos estudios plantean que existiría una relación entre actitudes docentes, aceptación o rechazo social en el aula con los estilos de crianza (Justicia et at., 2006; Cox, Jaramillo y Reimiers, 2006). Las dimensiones de soporte, control, afecto y apoyo de profesores y padres adquieren importancia central, constatándose efectos no deseados como el desarrollo de conductas de riesgo tanto en contextos de escasa supervisión como de excesivo control (Mestre, Tur, Samper, Nácher y Cortés, 2007; Estévez et al., 2008; Florenzano, et al., 2009; Isaza y Henao, 2012).

La funcionalidad, atmósfera e interacciones que se desarrollan en la familia contribuyen a dar sentido de realidad, formar actitudes y desarrollar comportamientos, por lo que la aparición de conductas negativas y desadaptativas en el aula son repercusión de inadecuados estilos parentales (Papalia, Wendkos y Duskin, 2005; Richaud De Minzi, 2005; Romero, Robles y Lorenzo, 2006; Oría, Peña e Inda, 2008; Isaza y Henao, 2012).

Por su parte, las habilidades sociales y democráticas evidenciadas en el aula se nutren de adecuadas dinámicas familiares y conductas de apoyos de parte de los padres; del mismo modo, una interacción continua en los establecimientos educativos puede acompañar a la familia en el desarrollo de modelos de conducta y aprendizaje efectivos para los estudiantes (Alcalay, Milicic y Torreti, 2005; Bernal, Estrada y Franco, 2006; Estévez et al., 2008; Martín-Quintana et al., 2009). Y, si bien se dispone de evidencia que respalda la implicancia familiar en el rendimiento académico, aquella también aporta significativamente a la conducta y estilo relacional de los alumnos (Lastre, López y Alcázar, 2018; Ibabe, 2016).

No obstante, los estilos de crianza no resumen toda la dinámica familiar, por cuanto inciden también factores de bienestar social y emocional, de salud y económicos en la forma en la que la familia inculca modelos de interacción social en sus hijos (Mestre et al, 2007; Ossa y Navarrete, 2011; Isaza y Henao, 2012).

Por ello, atendiendo a la importancia que tiene el vínculo familia-escuela en la generación de condiciones positivas para el aprendizaje y un buen clima áulico, este estudio aporta evidencias en torno a las actitudes y valoraciones que 
tienen los niños, niñas y adolescentes chilenos con respecto al soporte familiar y apoyo docente percibido como pilares de un desarrollo integral y la instalación de procesos educativos de calidad.

\section{Metodología}

\section{a. Instrumento}

Esta investigación hace uso de los resultados de la Primera Encuesta de DesarroIlo Humano en Niños, Niñas y Adolescentes (EDH-NNA), aplicada en Chile por el Programa de Naciones Unidas para el Desarrollo (PNUD) y el Consejo Nacional de la Infancia durante el año 2017. La encuesta tuvo por objeto proyectar la perspectiva de los NNA con respecto a su experiencia vital y la evaluación que hacen de las condiciones que la sociedad está generando para que esta etapa de sus vidas sea una experiencia positiva y satisfactoria.

Al respecto, esas opiniones servirían de insumo en la búsqueda de un nuevo trato de la sociedad chilena para con la niñez y la adolescencia, reconociéndoles como sujetos de Derecho a los cuales se les visibiliza, respeta y promueve su expresión.

El cuestionario, aplicado a jóvenes entre 12 y 17 años, bajo la modalidad de entrevista cara a cara asistida por computador (CAPI), se estructura en torno a 11 dimensiones, relevándose por su contingencia al bienestar subjetivo de los NNA las siguientes: Percepción de la educación y satisfacción escolar; Evaluación de la experiencia familiar y Satisfacción con vínculos significativos.

\section{b. Muestra}

Mediante un muestreo trietápico y probabilístico de estudiantes de $7^{\circ}$ básico a $3^{\circ}$ medio del territorio nacional, esta encuesta comprende un total de 3073 niños, niñas y adolescentes, distribuidos en 272 establecimientos educativos (municipales, particulares subvencionados y particulares pagados).

En su reseña técnica, el estudio reporta un margen de error de \pm 1.8 puntos porcentuales, un nivel de confianza del $95 \%$, una tasa de respuesta del $73,0 \%$ y de rechazo del $4 \%$. 
Tabla 1

Caracterización de la muestra (\%).

\begin{tabular}{lr|lr}
\hline Zona & & Sexo & \\
Urbana & 88,8 & Hombre & 52,9 \\
Rural & 11,2 & Mujer & 47,1 \\
\hline Rama educativa & & Nivel educativo & \\
Humanista & 92,4 & Básico & 66,4 \\
Técnico Profesional & 7,6 & Secundario & 33,6 \\
\hline Dependencia & & & \\
Municipal & 43,8 & & \\
Particular Subvencionado & 50,9 & & \\
Particular Pagado & 5,2 & & \\
\hline
\end{tabular}

Fuente: EDH-NNA, PNUD (2017). Elaboración propia.

En su mayor parte son estudiantes de zonas urbanas (88,8\%), de la rama Científico Humanista (92,4\%); un tercio cursan estudios Secundarios $(33,6 \%)$ con predominancia del sexo masculino $(52,9 \%)$ y de colegios Particulares $(56,1 \%)$.

\section{c. Procedimiento}

Este estudio explora los ítems correspondientes a las dimensiones de Percepción de la educación y satisfacción escolar (12 ítems), Evaluación de la experiencia familiar (12 ítems) y Satisfacción con vínculos significativos (3 ítems). Se analiza la percepción evaluativa de niños, niñas y adolescentes con respecto a la confianza que les brinda la familia, la escuela y los amigos; la percepción del soporte familiar y apoyo docente recibido; y la valoración que otorgan a la calidad de su proceso educativo, realizando inferencias y distinciones con base al nivel de enseñanza, sexo y tipo de establecimiento mediante pruebas estadísticas de ji cuadrado.

\section{Resultados}

Frente a la necesidad de recurrir por ayuda con ocasión de algún problema importante o preocupación, los NNA perciben mayor confianza en recibirla por parte de su familia $(88,2 \%)$ y sus amigos $(80,1 \%)$. La escuela como institución adquiere un rol comparativamente menos preponderante $(58,6 \%)$. 
La familia es la entidad de resguardo emocional y de cobijo por antonomasia, especialmente para los hombres $\left[X_{(1)}^{2}=47,676 ; p=0,000\right]$. Por su parte, ni el nivel educativo $\left[X^{2}{ }_{(1)}=0,316 ; p=0,574\right]$, ni el tipo de establecimiento $\left[X^{2}{ }_{(1)}=0,006\right.$; $\mathrm{p}=0,94]$ marcan diferencias estadísticamente significativas en el nivel de confianza declarado.

\section{Tabla 2}

Nivel de confianza acerca de recibir ayuda según nivel educativo, sexo y tipo de establecimiento (\%).

\begin{tabular}{lccccccc}
\hline \multirow{2}{*}{ General } & \multicolumn{2}{c}{ Nivel } & \multicolumn{2}{c}{ Sexo } & \multicolumn{2}{c}{ Tipo de establecimiento } \\
\cline { 3 - 8 } & & Básico & Medio & Hombre & Mujer & Municipal & Subvencionado \\
\hline Familia & 88,2 & 88,4 & 87,7 & 92,0 & 83,9 & 88,2 & 88,3 \\
\hline Escuela & 58,6 & 62,0 & 51,8 & 62,6 & 54,1 & 59,9 & 58,1 \\
\hline Amigos & 80,1 & 79,5 & 81,2 & 79,5 & 80,7 & 79,1 & 80,4 \\
\hline
\end{tabular}

Nota: Valores expresan porcentaje acumulado de las categorías de respuesta "Total Confianza" y "Bastante Confianza" frente a la pregunta "Si tuvieras un problema importante o una preocupación ¿Cuánta confianza tienes de que recibirás ayuda de...?

Fuente: EDH-NNA, PNUD (2017). Elaboración propia

A la institución escolar se le asigna una baja valoración en términos de confianza percibida, en particular para estudiantes mujeres $\left[X^{2}{ }_{(1)}=22,740 ; p=0,000\right]$ $y$ de enseñanza media $\left[X^{2}{ }_{(1)}=29,312 ; p=0,000\right]$. La dependencia administrativa del establecimiento educativo no reporta diferencias estadísticamente relevantes $\left[X^{2}{ }_{(1)}=0,906 ; p=0,341\right]$.

Por otro lado, la confianza percibida en los amigos como fuente de ayuda para contingencias o preocupaciones no evidencia significancia estadística en ninguna de las variables de segmentación [ $p>0,05]$. Para los niños, niñas y adolescentes la amistad adquiere alta preponderancia y es un factor de protección emocional con independencia del sexo, el tipo de establecimiento y nivel educativo en que se encuentren. 
Tabla 3

Apoyo familiar percibido que tienen los NNA según variables de segmentación.

\begin{tabular}{|c|c|c|c|c|}
\hline Ítems & $\%$ & Nivel & Sexo & Tipo \\
\hline 1. Desean saber qué estás haciendo & 86,7 & - & $>\mathrm{Hom}^{\star *}$ & - \\
\hline 2. Reconocen las cosas buenas que haces & 87,3 & $>\mathrm{Bas}^{\star *}$ & $>\mathrm{Hom}^{\star *}$ & - \\
\hline 3. Te preguntan cómo estás y cómo te sientes & 85,9 & $>$ Bas $^{*}$ & $>\mathrm{Hom}^{\star *}$ & $>$ Sub* \\
\hline $\begin{array}{l}\text { 4. Te animan y apoyan para que te vaya bien } \\
\text { en la escuela }\end{array}$ & 92,5 & $>$ Bas $^{*}$ & $>\mathrm{Hom}^{\star *}$ & - \\
\hline 5. Te dan libertad para tomar decisiones & 72,8 & - & $>\mathrm{Hom}^{\star \star}$ & $>$ Sub* \\
\hline 6. Confían en tu capacidad para elegir amigos & 83,9 & $>$ Bas $^{*}$ & $>\mathrm{Hom}^{* *}$ & - \\
\hline 7. Consideran tus ideas y opiniones & 76,2 & - & - & - \\
\hline 8. Confían en tu capacidad para lograr metas & 92,4 & - & $>\mathrm{Hom}^{\star *}$ & - \\
\hline 9. Te hacen sentir protegido y seguro & 93,8 & - & $>\mathrm{Hom}^{\star *}$ & - \\
\hline 10. Te hacen sentir querido & 92,1 & - & $>\mathrm{Hom}^{\star *}$ & - \\
\hline 11. Te apoyan en tus proyectos & 88,9 & - & $>\mathrm{Hom}^{\star *}$ & $>$ Sub* \\
\hline $\begin{array}{l}\text { 12. Te dicen que se sienten orgullosos de lo } \\
\text { que haces }\end{array}$ & 81,3 & $>$ Bas $^{* *}$ & $>\mathrm{Hom}^{\star *}$ & - \\
\hline
\end{tabular}

Nota: \% = Valores expresan porcentaje acumulado de las categorías de respuesta "Siempre" y "Casi Siempre a nivel general; ${ }^{*}=$ significativo al $95 \%$ nivel confianza; ${ }^{* *}=$ significativo al $99 \%$ de nivel de confianza; - = No se reporta significancia; >= establece la categoría predominante en el contraste; Bas= Nivel educativo básico; Hom= Hombre; Sub= Colegio Particular Subvencionado.

Fuente: EDH-NNA, PNUD (2017). Elaboración propia.

Prácticamente la totalidad de los NNA manifiesta buena evaluación de su familia como soporte emocional y afectivo, declarando que es un lugar donde se sienten protegidos y seguros (93,8\%), queridos $(92,1 \%)$, apoyan sus proyectos $(88,9 \%)$, se interesan por saber qué están haciendo $(86,7 \%)$, les reconocen las cosas buenas que hacen $(87,3 \%)$ y les animan y apoyan para que les vaya bien en la escuela $(92,5 \%)$.

En el contraste inferencial, se constatan diferencias estadísticamente significativas por sexo en prácticamente todos los ítems analizados. De esa manera, los hombres reportan mayores niveles de confianza hacia su familia $[p<0,01]$. 
Con respecto al nivel educativo, los estudiantes de enseñanza básica expresan mayor apoyo de su grupo familiar, aunque estas diferencias no son significativas en todos los ítems [i2 e i12 $p<0,01 ;$ i3, i4 e i6 $p<0,05]$.

Mucho más homogénea es la confianza de apoyo percibido con base al tipo de establecimiento, constatándose contrastes significativos en los ítems vinculados al apoyo en proyectos, preocupación por su estado de ánimo y libertad para la toma de decisiones [i3, i5 e i11 $p<0,05]$, siempre a favor de los estudiantes de colegios Particulares Subvencionados.

\section{Tabla 4}

Apoyo Docente Percibido que tienen los NNA según variables de segmentación.

\begin{tabular}{lcccc}
\hline & $\%$ & Nivel & Sexo & Tipo \\
\hline 1. Te tratan con respeto y amabilidad & 94,9 & - & - & - \\
\hline 2. Te motivan a seguir aprendiendo & 93,8 & $>$ Bas $^{\star *}$ & - & - \\
\hline 3. Confían en tus capacidades & 91,3 & - & - & - \\
\hline 4. Permiten que expreses tus opiniones & 92,0 & - & - & - \\
\hline 5. Te ha insultado o humillado & 6,2 & $>$ Med $^{*}$ & - & - \\
\hline 6. Consideran tus aportes o sugerencias & 88,2 & - & - & - \\
\hline
\end{tabular}

Nota: $\%$ = Valores expresan porcentaje acumulado de las categorías de respuesta "Siempre" y "Casi Siempre" a nivel general; ${ }^{*}=$ significativo al $95 \%$ nivel confianza; ${ }^{* *}=$ significativo al $99 \%$ de nivel de confianza; - = No se reporta significancia; >= establece la categoría predominante en el contraste; Bas= Nivel educativo básico; Med= Nivel educativo Media; Hom= Hombre.

Fuente: EDH-NNA, PNUD (2017). Elaboración propia.

Por su parte, los estudiantes manifiestan altos niveles de apoyo docente en su proceso de aprendizaje. También, informan que siempre o casi siempre el profesor los trata con respeto $(94,9 \%)$, los motiva a seguir aprendiendo (93,8\%), le permite expresar opiniones (92,0\%) y confía en las capacidades del alumno (91,3\%). En todos estos ítems no se observan diferencias significativas según variables de segmentación consignadas, a excepción de la sentencia que indaga con respecto a la motivación para el aprendizaje, la cual es reportada con mayor prevalencia por estudiantes de ciclo básico [X2(4)=41,912; $p=0,000]$.

Un 6,2\% de estudiantes informa que "Siempre o casi siempre" ha sido objeto de insultos y humillaciones por parte del profesor, cifra que se incrementa al $11,0 \%$ 
al agregar la categoría de respuesta "Algunas veces". Percepción que es más acentuada en estudiantes de enseñanza media $\left[X^{2}{ }_{(4)}=11,892 ; p=0,018\right]$.

\section{Tabla 5}

Valoración de la Calidad Educativa que hacen NNA según variables de segmentación.

\begin{tabular}{lcccc}
\hline & $\%$ & Nivel & Sexo & Tipo \\
\hline 1. Entregan buena educación & 95,5 & - & - & - \\
\hline 2. Posibilidad de elegir actividades & 87,7 & - & $>$ Hom $^{\star *}$ & $>$ Mun $^{\star *}$ \\
\hline 3. Recursos necesarios & 93,2 & $>$ Bas $^{\star *}$ & - & $>$ Mun $^{*}$ \\
\hline 4. Incentiva a valorar la diversidad & 93,4 & $>$ Bas $^{*}$ & $>$ Hom $^{\star *}$ & - \\
\hline 5. Incentiva participación y organización & 92,5 & - & - & - \\
\hline 6. Deseas cambiar de escuela & 27,8 & $>$ Bas $^{\star *}$ & $>$ Mujer $^{* *}$ & $>$ Sub $^{\star *}$ \\
\hline
\end{tabular}

Nota: \%= Valores expresan porcentaje acumulado de las categorías de respuesta "Muy de acuerdo" y "De acuerdo" a nivel general; * = significativo al $95 \%$ nivel confianza; **= significativo al $99 \%$ de nivel de confianza; - = No se reporta significancia; >= establece la categoría predominante en el contraste; Bas= Nivel educativo básico; Hom= Hombre; Sub= Colegio Particular Subvencionado; Mun= Colegio Municipal.

Fuente: EDH-NNA, PNUD (2017). Elaboración propia.

Con respecto a la valoración que hacen de la calidad educativa de su establecimiento, los estudiantes consideran que: entrega buena educación (95,5\%), dispone de los recursos necesarios $(93,2 \%)$ especialmente en el caso de estudiantes de enseñanza básica $\left[X_{(1)}^{2}=11,901 ; p=0,001\right]$ y de escuelas Municipales $\left[X^{2}{ }_{(1)}=5,452 ; p=0,020\right]$. Además, señalan en alta proporción que se incentiva la participación $(92,5 \%)$ y diversidad $(93,4 \%)$, siendo especialmente elevada en estudiantes varones $\left[X^{2}{ }_{(1)}=10,215 ; p=0,001\right]$ y en escuelas básicas $\left[X^{2}(1)=5,872 ; p=0,015\right]$.

Además, la posibilidad de elegir actividades, aún siendo alta $(87,7 \%)$, se expresa con mayor intensidad en hombres $\left[X^{2}{ }_{(1)}=9,206 ; p=0,002\right]$ y escuelas municipales $\left[X^{2}{ }_{(1)}=24,113 ; p=0,000\right]$.

En este apartado llama la atención la alta proporción de estudiantes que manifiestan su deseo de cambiarse de escuela $(27,8 \%)$, particularmente elevado en el caso de estudiantes de Enseñanza Básica [ $\left.X^{2}{ }_{(1)}=10,522 ; p=0,001\right]$, Mujeres $\left[X^{2}{ }_{(1)}=8,224 ; p=0,004\right]$ y Particulares Subvencionados $\left[X^{2}{ }_{(1)}=6,708 ; p=0,009\right]$. 


\section{Discusión de resultados}

Desde la primera infancia, como seres humanos, habitamos en contextos sociales complejos. Nuestra naturaleza intrínsecamente social nos empuja a desarrollar una serie de habilidades emocionales que nos permitan reconocer y comprender a los otros (López, Arán y Richaud de Minzi, 2014); capacidad sin la cual no podríamos entablar relaciones de amistad, confianza o solidaridad, necesarias para vivir satisfactoriamente (Watt, 2007; Richaud de Minzi, 2017). Esta cuestión, procedente de los avances en la comprensión y búsqueda del bienestar, es clave para dilucidar los desafíos contemporáneos en materia de desarrollo humano, el cual se agrega a la preocupación por asegurar los medios para que la población viva de forma ética y materialmente bien (Buelna, Gutiérrez y Ávila, 2010); además, cobra importancia la necesidad de generar las condiciones sociales óptimas para que las personas se sientan satisfechas, tanto con su vida como con la sociedad donde la despliegan (PNUD, 2012).

En tal sentido, es indispensable para el Estado chileno avanzar política y progresivamente en la creación de normativas e instituciones que, por un lado, garanticen la aplicación de los principios que estableció la Convención sobre los Derechos del Niño (CDN) y su protección (SENAME, 2017) y, por otro, instalar un nuevo paradigma en la aceptación y visibilización de su rol como actor social, más dirigido a sus potencialidades y anhelos (Gobierno de Chile, 2015). Por ello, y como parte de la Política Nacional de Niñez y Adolescencia 2015-2025, se realizó la Primera Encuesta de Desarrollo Humano en Niños, Niñas y Adolescentes (NNA), dentro de la cual destacamos los siguientes resultados de análisis.

La confianza, hecho básico de la vida social dotado de su propia sustancia, lógica y estructura (Luhmann, 1996; Coleman, 2011; Callejo, 2017), se instala como un sentimiento de cariz compleja entre los estudiantes chilenos, estrechamente vinculada al tipo de institución y relaciones que allí se albergan.

Siendo la familia el sitio por excelencia donde las personas encontramos soporte, contención y estímulos (Parsons, 1970; Estévez et al., 2008; Fawaz, Soto y Zícavo, 2014; Murueta, 2014), es para los niños, niñas y adolescentes la entidad en la que más confian para recibir ayuda, dando cuenta de su capacidad intrínseca para la promoción de conductas prosociales y de afrontamiento en los diversos ámbitos de interacción, incluyendo aquellos relacionados con la convivencia y rendimiento escolar (García Núñez, 2005; Isaza y Henao, 2012). 
Al respecto, en la medida en la que los NNA estudiantes perciben que sus opiniones son respetadas por sus padres y señalan sentirse orgullosos, los apoyan y reconocen las cosas buenas que hacen, estos ven fortalecidas sus herramientas constructivas para enfrentar situaciones adversas de manera adaptativa, como resultado de un resguardo afectivo y anímico (Estévez et al., 2008).

Incluso, a pesar del impacto que ha tenido la vorágine de cambios asociados al trabajo, inmersión laboral femenina e irrupción de las nuevas tecnologías sobre las formas y modos de comunicación interpersonal, la familia mantiene su impronta como entidad de soporte emocional y educativo para los hijos.

Por otro lado, la escuela como objeto de confianza comporta una valoración significativamente menor en comparación a la familia y los amigos. Para los NNA estudiantes, la institución educativa cumple una función eminentemente pedagógica, con reglas, normas y procedimientos que la hacen tomar distancia de otros roles, como ser confidente de las problemáticas y preocupaciones del alumnado. Es por tanto una representación simbólica de la escuela como entidad abstracta e impersonal, escindida de las aulas, donde es el profesor quien adquiere un rol preponderante como figura de autoridad y vinculación afectiva.

Una explicación abierta reside en la configuración de los climas de convivencia escolar, los cuales contienen y perfilan la interacción y relación entre los diferentes actores de la comunidad educativa. Además, los estudios recientes han puesto en tela de juicio la armonia y neutralidad como características sine quanon de los espacios educativos, denunciando la necesaria atención en la frecuencia con la que suceden episodios de violencia física, verbal y emocional al interior del colegio (Lomas, 2007; Tijmes, 2012; Treviño, Place y Gempp, 2012; López de Mesa et al., 2013).

La confianza y vínculos significativos que los alumnos forjan con la escuela es relevante, por cuanto se observa la existencia de una interacción entre clima de aula y conductas positivas, pues las habilidades prosociales y de amistad actuarían de freno ante manifestaciones agresivas y de inestabilidad emocional (Mestre, Frías y Samper, 2004; Sánchez, Rivas y Trianés, 2006; Treviño, Place y Gempp, 2012). Asimismo, la presencia de conductas disruptivas en las relaciones con pares y profesores deteriora el ambiente propicio para el aprendizaje. En este contexto, tanto estudiantes inhibidos, tímidos 0 aislados, como alumnos agresivos, impulsivos o violentos, son sujetos en riesgo que presentan problemas interpersonales 0 de desajuste social que deben ser atendidos por la institución 
escolar, en el entendido que parte de las dificultades conductuales de niños y adolescentes pasan por las imprecisiones, ausencias, frustraciones y vacíos que experimentan numerosos padres al verse inmersos en tantos cambios vertiginosos que se suceden en la sociedad actual.

En todo caso, en la medida en la que la escuela es percibida como una entidad acogedora y digna de confianza, mejoran las condiciones del clima de convivencia, elemento sustancial para el desarrollo del aprendizaje y de las habilidades sociales y emocionales (Blanco, 2008; Paiva y Saavedra, 2014; Gazmuri, Manzi y Paredes, 2015).

Por otro lado, al descomponer el apoyo familiar percibido, se observan altas prevalencias en cada uno de sus ítems, lo que reporta una muy buena percepción que tienen niños, niñas y adolescentes del trato parental recibido, en particular, hombres y alumnos de enseñanza básica. Esto es relevante por cuanto la relación que se establece con la familia es un elemento imprescindible para el desarrollo óptimo de las capacidades sociales y de afrontamiento (Steinberg y Lerner, 2004; Armstrong, Birnie-Lefcovitch, y Ungar, 2005; Florenzano et al., 2009; Isaza y Henao, 2012), situación especialmente trascendental cuando los niños son más pequeños y se sienten más cercanos a los atributos identitarios del grupo familiar. El tipo de interacción familiar y la evaluación que los hijos hacen del papel de los padres tienen un efecto positivo en la calidad de vida y el bienestar subjetivo (Rodríguez, Verdugo y Cruz, 2008), el cual no se construye únicamente con base a la conducta objetiva manifestada por los padres, sino que, además, es influenciado por la valoración que el niño hace de ella (Bleichmar, 2004).

Si bien las cifras hacen parecer que la autonomía de los jóvenes está comprometida, exhibiendo un nivel de confianza reportada inferior a los otros ítems que componen esta dimensión (i5, i7, i12), se considera que, en razón de la edad en la que se encuentran, no pasa por ellos la toma de decisiones personales o familiares de envergadura, lo que comporta un sentimiento de frustración con respecto a la libertad de elegir los aspectos sobre los cuales los padres tienen tuición y responsabilidad (Estévez et al., 2008). El estadio de moratoria moral, dependencia política y económica en el que se encuentran supone por parte de los padres 0 adultos responsables tomar control sobre determinadas acciones de sus hijos como medida de precaución o disciplina. Esta preocupación es parte del rol parental educativo que otorga a la familia un referente de sentido (Romera, 2003), donde la riqueza de las condiciones y objetivos del proyecto de vida grupal 
se relacionan con el éxito de sus miembros, y son causantes del bienestar físico y emocional de los hijos (Mingo y Escudero, 2008; Isaza y Henao, 2012).

Por otro lado, los padres con autoridad son más eficaces en la enseñanza de conductas y valores sociales que los autoritarios o permisivos (Baumrind, 1966), al hacer mejor uso del afecto y refuerzo positivo, lo que implica poner límites consistentes y estar más comprometidos. No obstante, cuando estos actos de responsabilidad parental e involucramiento superan los límites del ámbito personal y se entrometen en la definición de actitudes o comportamientos que forman parte de lo que los jóvenes consideran sustancial a su identidad, pueden constituirse como un factor de riesgo del desarrollo individual (Isaza y Henao, 2012).

De esa manera, el control patológico, las relaciones cargadas de hostilidad, crítica y rigidez, producen persistentes malas relaciones e infunden ansiedad en los hijos, reduciendo su capacidad para enfrentar situaciones adversas (Mestre, et al, 2007, 2003; Richaud de Minzi, 2006).

Ahora bien, siguiendo los datos reportados en la Tabla 4, las altas puntuaciones exhibidas en los ítems referidos al involucramiento docente percibido dan cuenta de la existencia de adecuados contextos interaccionales en la escuela para promover el aprendizaje e instalar competencias prosociales. Situación de relevancia toda vez que el clima escolar y áulico, en particular, la calidad del vínculo profesor-alumno, se relaciona con la motivación para el aprendizaje, el rendimiento académico, la retención escolar, el bienestar físico y emocional de los estudiantes (Blanco, 2006; Valenzuela, 2007; Jiménez, 2009).

En la medida que los alumnos se sienten aceptados, respetados, escuchados y valorados en sus opiniones e inquietudes, mejora su bienestar emocional, tributando a su predisposición actitudinal para con el aprendizaje y el clima áulico y escolar (Meehan, Hughes y Cavell, 2003). Además, el cómo se realizan las clases y el estilo pedagógico que utiliza el profesor impactan en la percepción de satisfacción y significado que el estudiante desarrolla hacia el aprendizaje, partiendo por la capacidad que ellos perciben en la figura docente para establecer un buen clima áulico, ser anímico en lugar de punitivo, utilizar estrategias didácticas y responder atentamente a las inquietudes. En sentido contrario, la predominancia de autoritarismo, prejuicio, competitividad y escaso apoyo docente, configura entornos inhibidores para el desarrollo de habilidades comunicativas, de negociación y empatía. 
Por otro lado, el concepto de calidad de la educación ha estado fuertemente ligado a aspectos curriculares, como son las habilidades lectoras y matemáticas en los estudiantes. No obstante, y pese a existir consenso en que ello resulta fundamental para el avance e instalación de destrezas cognitivas complejas, cada vez con mayor recurrencia se instala en los círculos académicos y en la práctica docente la necesidad de considerar aspectos contextuales del proceso de enseñanza-aprendizaje en su estimación evaluativa. Por tanto, la alta valoración que los estudiantes hacen de la calidad educativa (Tabla 5) supone la existencia de un buen clima escolar y áulico, y de un ambiente organizado y seguro, donde se promueve el trato respetuoso y se fortalece el vínculo entre los miembros de la comunidad escolar, aumentando así la capacidad para la resolución constructiva de conflictos (López de Mesa et al., 2013).

Asimismo, los mayores niveles de satisfacción con la escuela se correlacionan con un mejor clima áulico y rendimiento académico, en tanto, se disfruta de lo aprendido y considera gratificante, repercutiendo en sus logros tanto a nivel personal como social (Paneiva, Bakker y Rubiales, 2018; Barr, 2016; Rentería et al., 2013; Treviño, Place y Gempp, 2012).

En todo caso, la consecución del objetivo de generar aprendizajes cognitivos y actitudinales con eficiencia es tanto cuestión de disponibilidad de recursos necesarios y suficientes como de capacidad institucional para atender la diversidad e incentivar la participación.

Es importante destacar que, si bien un buen clima escolar como el reportado en los datos afianza el sentido de pertenencia hacia la institución escolar, llama la atención la alta proporción de estudiantes que desean cambiarse de establecimiento. Esta disonancia observada podría ser, por un lado, expresión de individualismo y falta de sentido de pertenencia incentivada por un sistema educativo que promueve la libre elección y la búsqueda de mejores proyecciones educativas; y por otro, un sesgo de composición muestral al contemplar alumnos en tránsito a la enseñanza media ( $8^{\circ}$ Básico), situación que condiciona el sentido del ítem por cuanto la continuidad de la trayectoria educativa deriva en la generalidad de los casos en cambio de establecimiento. 


\section{Conclusiones}

En la actualidad, el análisis del clima escolar y áulico ha migrado sus intereses del aspecto físico o estructural del colegio y sala de clases hacia modelos de comprensión más complejos, donde las relaciones psicosociales entre estudiantes, profesores y miembros de la familia adquieren centralidad. Lo anterior, bajo la premisa de que: un clima positivo favorece el desarrollo cognitivo y emocional, mejora los niveles de rendimiento académico, crea oportunidades de aprendizaje significativo y favorece el desarrollo de habilidades cognitivas.

Los resultados aquí expuestos responden a la inquietud por describir y visibilizar la relación que existe entre la confianza que los NNA sienten hacia la escuela y su familia, la percepción que tienen del apoyo familiar, el apoyo docente que reciben durante las clases y el valor que otorgan a la experiencia educativa que les entrega el establecimiento.

Al respecto, destacamos que en lo que a niños, niñas y adolescentes chilenos de $8^{\circ}$ Básico y $2^{\circ}$ Medio se refiere, la institución que mejor confianza proyecta es la familia, secundada por las relaciones interpersonales de amistad y, visiblemente por detrás, la escuela. Esto es señal de la relevancia que tiene la proximidad y cercanía en las relaciones para la definición de importancia, pues los padres y amigos al estar constantemente interactuando con los jóvenes adquieren altas valoraciones en compañía, apoyo y seguridad. Ello explicaría, también, la posición que tiene el docente dentro del aula y sus calificaciones por parte de los estudiantes, las cuales son mayores a las otorgadas a la institución y sería extensión de la sensación de que la escuela, por un lado, es algo jerárquico de difícil acceso, distinto al profesor, quien, a pesar de formar parte de la estructura escolar, está en las aulas compartiendo y recreando un espacio de cotidianeidad.

Sobre el segundo eje de discusión, encontramos que el apoyo familiar es imprescindible para los sentimientos de seguridad y respaldo en los estudiantes encuestados, quienes aprecian significativamente el cobijo emocional y aliento personal y académico que allí reciben. No obstante, los componentes de libertad y autonomía se perciben restringidos. Ello no es necesariamente negativo, en tanto, es consustancial a las etapas de infancia y adolescencia, y a la existencia de modelos de crianza protectores y de regulación conductual. Los mecanismos y dispositivos de control parental se constituyen en riesgos para la salud emocional, física y social de los hijos cuando siguen derroteros de maltrato, agresión 0 privación excesiva. 
Por otra parte, el apoyo que los profesores entregan en la sala de clases es reportado por los estudiantes como satisfactorio, dado que el respeto, motivación y estímulos hacia el aprendizaje significativo priman por sobre conductas pedagógicas punitivas o sancionatorias.

Igualmente, los alumnos manifiestan gran conformidad con la calidad de la educación que reciben, los recursos disponibles y el incentivo que se hace a la diversidad y participación. También, destaca una alta valoración de las condiciones para el aprendizaje y convivencia que cohabitan con una elevada proporción de estudiantes que expresan el deseo por cambiarse de establecimiento. Disonancia posiblemente explicada por un reducido sentido de pertenencia en contextos de libre elección, como por sesgos de muestra que condicionan el sentido del ítem en estudiantes de ciclo básico, quienes migran para prolongar su trayectoria escolar.

En suma, este artículo pretende aportar evidencias al emergente campo del clima escolar y áulico como factor de relevancia para la estimación de la calidad educativa en Chile y el estado del Desarrollo Humano en población escolar. Es imprescindible, según lo expuesto, estrechar los vínculos de cooperación entre los agentes educativos y familiares, en especial docentes y padres.

La percepción positiva que los estudiantes desarrollan y manifiestan de su familia y escuela constituyen la sustancia para su sensación de bienestar y satisfacción individual, por lo que futuras estrategias de mejoramiento educativo tienen que hacerse cargo de profundizar la relación entre ambas instituciones. Quizás, el mayor desafío reside en la disponibilidad que los padres y apoderados tienen para responder a las demandas educativas, mientras se desenvuelven en contextos laborales y sociales también complejos y exigentes. 


\section{REFERENCIAS BIBLIOGRÁFICAS}

ALCALAY, L., MILICIC, N., Y TORRETTI, A. (2005). "Alianza efectiva familiaescuela: un programa audiovisual para padres". Psykhe , 149-161.

AMBROSE, S., BRIDGES, M., DIPIETRO, M., Y LOVETT, M. (2010). How learning works; Seven research-based principles for smart teaching. San Francisco: Jossey Bass.

ARMSTRONG, M., BIRNIE-LEFCOVICH, S., Y UNGAR, M. (2005). "Pathways bet-ween social support, quality of parenting and child resilience: $A$ transactional model". Journal of Family and Child Studies, 14(2), 269281.

ARON, A. M., MILICIC, N., Y ARMIJO, I. (2012). "Clima social escolar: una escala de evaluación - Escala de Clima Social Escolar, ECLIS”. Universitas Psychologica, 11(3), 803-813.

BARR, J. (2016). Developing a Positive Classroom Climate. Paper 61. The IDEA Center.

BAUMRIND, D. (1966). "Effects of authoritative parental control on child behavior". Child Development, 37(4), 887-907.

BAZÁN, A., SÁNCHEZ, B., Y CASTAÑEDA, S. (2007). "Relación estructural entre apoyo familiar, nivel educativo de los padres, características del maestro y desempeño en lengua escrita". Revista Mexicana de Investigación Educativa, 33(12), 701-729.

BERNAL, R. E., ARBELÁEZ, F., Y CRISTINA, M. (2006). "Ambiente humano: un enfoque para la formación de ciudadanos". Educación y Educadores, 9(1), 135-145.

BLANCO, R. (2006). "La equidad y la inclusión social: uno de los desafíos y la escuela hoy". Revista Iberoamericana sobre Calidad, Eficacia y Cambio en Educación, 4(3), 1-15.

BLANCO, R. (2008). "Construyendo las bases de la inclusión y la calidad de la educación en la primera infancia”. Revista de educación (347), 33-54. 
BLEICHMAR, E. (2004). "Modelos interactivos entre la genética de la conducta y la parentalización". Aperturas psicoanalíticas: Revista de psicoanálisis, 17(5).

BUELNA, M., GUTIERREZ, L., Y ÁVILA, S. (2010). "Ilustración, progreso y bienestar: crítica al historicismo". Análisis Económico, 25(60), 303-329.

CABALLERO, M. (2010). "Convivencia escolar. Un estudio sobre buenas prácticas". Revista paz y conflictos(3), 154-168.

CALLEJO, J. (2017). "La cultura de la confianza en tiempos de crisis: análisis de los discursos". Revista Española de Sociología, 26(2), 185-20.

CASSINDA, M., ANGULO, L., Y GUERRA, V. (2017). "Características de la expresión emocional en los escolares primarios y su manejo desde la perspectiva del personal docente-educativo". Revista Electrónica Educare, 1(1).

CAVA, M., Y MUSITU, G. (2002). La convivencia en las escuelas. Barcelona: Paidós.

CÉSPEDES, A. (2008). Educar las emociones, educar para la vida. Santiago de Chile: Ed. B S.A.

COLEMAN, J. (2011). Fundamentos de teoría social. Madrid: Centro de Investigaciones Sociológicas.

COX, C., JARAMILLO, R., Y REIMERS, F. (2006). Educar para la ciudadanía y la democracia en las Américas: Una agenda para la acción. Inter-American Development Bank.

DÍAZ, K., Y OSUNA, C. (2017). "Contexto sociofamiliar en jóvenes en situación de abandono escolar en educación media superior Un estudio de caso". Revista Perfiles Educativos, 39(158).

ESTÉVEZ, E., MURGUI, S., MUSITU, G., Y MORENO, D. (2008). "Clima familiar, clima escolar y satisfación con la vida en los adolescentes". Revista Mexicana de Psicología, 25(1), 119-128. 
FAWAZ, J., SOTO, P., Y ZíCAVO, N. (Edits.). (2014). Re-significando la familia en América Latina: entre imágenes y realidades. Chillán: Ediciones Universidad del Bío-Bío.

FLORENZANO, R., VALDÉS, M., CÁCERES, E., CASASSUS, M., SANDOVAL, A., SANTANDER, S., Y CALDERÓN, S. (2009). "Percepción de la relación parental entre adolescentes mayores y menores de 15 años". Revista chilena de pediatría, 80(6), 520-527.

GARCÍA NUÑEZ, C. (2005). "Habilidades sociales, clima social familiar y rendimiento académico en estudiantes universitarios". Liberabit. Revista de Psicología, 63-74.

GAZMURI, C., MANZI, J., Y PAREDES, R. (2015). "Disciplina, clima y desempeño escolar en Chile". Revista CEPAL(115), 116-128. Recuperado de: http://200.9.3.98/bitstream/handle/11362/37833/ REV115ManziParedes_es.pdf?sequence=1yisAllowed =y

GÁZQUEZ, J., PÉREZ, M. C., Y CARRIÓN, J. (2011). "Clima escolar y resolución de conflictos según el alumnado: un estudio europeo". Revista de Psicodidáctica, 16(1), 39-58.

GILBERT, J. (2010). Introducción a la sociología. Santiago: LOM.

GOBIERNO. (2015). Política Nacional de Niñez y Adolescencia 2015-2025: Sistema integral de garantías de derechos de la niñez y la adolescencia. Santiago de Chile: Consejo Nacional de la Infancia.

GUBBINS, V., TIRADO, V., Y MARCHANT, V. (2017). "Disposiciones docentes hacia la relación con apoderados en un establecimiento educacional privado en Chile: estudio de caso". Revista Perfiles Educativos, 39(155).

IBABE, I., Y BENTLER, M. (2016). "The contribution of family relationships to childto-parent violence". Journal of Family Violence, 31(2), 259-269.

ISAZA, L., Y HENAO, G. (2012). "Influencia del clima sociofamiliar y estilos de interacción parental sobre el desarrollo de habilidades sociales en niños y niñas". Persona: Revista de la Facultad de Psicología(15), 253-271. 
JIMÉNEZ, M. I. (2009). "Inteligencia emocional y rendimiento escolar: estado actual de la cuestión". Revista latinoamericana de Psicología, 41(1), 6979.

JUSTICIA, F., BENIITEZ, J. L., PICHARDO, M., FERNÁNDEZ, E., GARCÍA, T., Y FERNÁNDEZ, M. (2006). "Aproximación a un nuevo modelo explicativo del comportamiento antisocial". Electronic Journal of Research in Educational Psychology, 4(9).

LASTRE, K., LÓPEZ, L., Y ALCÁZAR, C. (2018). "Relación entre apoyo familiar y el rendimiento académico en estudiantes colombianos de educación primaria". Psicogente, 21(39), 102-115.

LEWIN, K. (1939). "Field theory and experiment in social psychology: concepts and methods". American journal of sociologý, 44(6), 868-896.

LILA, M., BUELGA, S., Y MUSITU, S. (2006). Las relaciones entre padres e hijos en la adolescencia. Madrid: Pirámide.

LOMAS, C. (2007). "¿La escuela es un infierno? Violencia escolar y construcción cultural de la masculinidad". Revista de Educación(342), 83-101.

LÓPEZ DE MESA, C., CARVAJAL, C., SOTO, M. F., Y URREA, P. (2013). "Factores asociados a la convivencia escolar en adolescentes". Educación y Educadores, 16(3), 383-410.

LÓPEZ, M., ARÁN, V., Y RICHAUD DE MINZI, M. (2014). "Empatía: algunos debates en torno al concepto". Avances de Psicología Latinoamericana, $32,37-51$.

LUHMANN, N. (1996). Confianza. Barcelona: Anthropos.

MARTíN, J., ALEMÁN, J., MARCHENA, R., Y SANTANA, R. (2015). "El contexto familiar del alumnado en riesgo de abandono escolar temprano según la tipología familiar". Revista de Curriculum y Formación del Profesorado, 3(19), 245-266. 
MARTÍN-QUINTANA, J., MÁIQUEZ CHAVES, M., LÓPEZ, R., JOSÉ, M., BYME, S., RODRÍGUEZ, B., Y RODRÍGUEZ, G. (2009). "Programas de educación parental”. Psychosocial Intervention, 18(2), 121-133.

MEEHAN, B., HUGHES, J., Y CAVELL, T. (2003). "Teacher-student relationships as compensatory resources for agressive children". Child development, 74, 1145-1157.

MESTRE, M., FRÍAS, M., Y SAMPER, P. (2004). "Personalidad y contexto familiar como factores predictores de la disposición prosocial y antisocial de los adolescentes". Revista latinoamericana de psicología, 36(3), 445 - 457.

MESTRE, M., TUR, A., SAMPER, P., NÁCHER, M., Y CORTÉS, M. (2007). "Estilos de crianza en la adolescencia y su relación con el comportamiento prosocial". Revista Latinoamericana de Psicología, 39(2), 211-225.

MILLER, A., Y CUNNINGHAM, K. (2011). "Classroom environment". Retrieved, 30(7).

MINGO, M., Y ESCUDERO, A. (2008). "Calidad de vida de los menores con discapacidad. Análisis de la situación en España desde la inclusión de los menores". En CIAP/UNED, Los menores con discapacidad en España (págs. 201-330). Madrid: Cinca.

MOOS, R. (1974). The social climate scale: an overview. Palo Alto: Consulting Psichologist Press.

MURUETA, M. (2014). "Las etapas del ciclo de vida familiar en la Teoría de la Praxis: riesgos y posibilidades". En J. Fawaz, P. Soto, y N. Zícavo (Edits.), Re-significando la familia en América Latina: entre imágenes y realidades (págs. 19-34). Chillán: Ediciones Universidad del Bío-Bío.

OSSA, C., Y NAVARRETE, L. (2011). Estilos de crianza y calidad de vida en padres de preadolescentes que presentan conductas disruptivas en el aula. Chillán: Tesis de Magíster en Familia. 
PAIVA, F., Y SAAVEDRA, F. (2014). "Clima social escolar y rendimiento escolar: Escenarios vinculados en la educación". (Tesis de pregrado). Universidad del Bío-Bío, Chillán, Chile. Recuperado de: http://repobib. ubiobio.cl/jspui/handle/123456789/232

PANEIVA, J. P., BAKKER, L., Y RUBIALES, J. (2018). "Climaáulico. Características socio-emocionales del contexto de enseñanza y aprendizaje". Educación y Ciencia, 7(49), 55-64.

PAPALIA, D., WENDKOS, S., Y DUSKIN, R. (2005). Psicología del desarrollo de la infancia a la adolescencia. México: McGraw-Hill.

PARSONS, T. (1970). La estructura social de la familia. Barcelona: Península .

PNUD. (2012). Informe de Desarrollo Humano en Chile. Bienestar subjetivo: el desafío de repensar el desarrollo. Santiago de Chile: Programa de las Naciones Unidas para el Desarrollo.

RENTERÍA, M., GONZÁLEZ, E., GARCÍA, M. T., Y MACÍAS, M. (2013). "El clima aulico y los factores que le afectan". Revista Iberoamericana para la Investigación y el Desarrollo Educativo(10), Recuperado de http://ride. org.mx/1-11/index.php/RIDESECUNDARIO/article/viewFile/262/257

RICHAUD DE MINZI, M. (2005). "Estilos parentales y estrategias de afrontamiento en niños". Revista latinoamericana de Psicología, 37(1).

RICHAUD, M. C. (2017). "Algunos aportes sobre la importancia de la empatía y la prosocialidad en el desarrollo humano". Revista Mexicana de Investigación en Psicología, 6(2), 171-176.

RODRÍGUEZ, A., VERDUGO, M., Y CRUZ, M. (2008). "Calidad de vida familiar y apoyos para los progenitores de personas con discapacidad intelectual en proceso de envejecimiento". Siglo Cero, 39(3).

RODRíGUEZ, N. (2004). "El clima escolar". Revista Digital de Investigación y Educación, 7(3), 2-12.

ROMERA, I. (2003). "Calidad de vida en el contexto familiar: dimensiones e implicaciones políticas". Psychosocial Intervention, 12. 
ROMERO, E., ROBLES, Z., Y LORENZO, E. (2006). "Prácticas parentales, atmósfera familiar y problemas de conducta externalizante en la infancia". Revista de Psiquiatría de la Facultad de Medicina de Barcelona, 33(2), 84-92.

SÁNCHEZ, A., RIVAS, M., Y TRIANES, M. (2006). "Eficacia de un programa de intervención para la mejora del clima escolar: algunos resultados". Electronic Journal of Research in Educational Psychology .

SENAME. (01-11-2017 de Noviembre de 2017). Ministerio de Justicia y Derechos Humanos. Obtenido de http://www.sename.cl/web/marco-legal-leyresponsabilidad-penal-adolescente/

STEINBERG, L., Y RICHARD, L. (2004). Handbook of adolescent psychology. New Jersey: John Wiley y Sons .

TIJMES, C. (2012). "Violencia y clima escolar en establecimientos educacionales en contextos de alta vulnerabilidad social de Santiago de Chile". Psykhe, 21(2), 105-117. doi: http://dx.doi.org/10.7764/psykhe.21.2.548

TORÍO, S., PEÑA, J., Y INDA, M. (2008). "Estilos de educación familiar". Psicothema, 2(1), 62-70.

TREVIÑO, E., PLACE, K., Y GEMPP, R. (2012). Análisis del clima escolar: poderoso factor que explica el aprendizaje en América Latina y el Caribe. Santiago: Oficina Regional de Educación para América Latina y el Caribe. OREALC/ UNESCO.

VALENZUELA, J. (2007). "Más allá de la tarea: pistas para una redifinición del concepto de motivación escolar". Educacao e pesquisa, 33(3), 409-426.

WATT, D. (2007). "Toward a neuroscience of empathy: Integrating affective and cognitive perspectives". Neuropsychoanalysis, 9, 119-140. 